Article

\title{
Risky Multi-Attribute Decision-Making Method Based on the Interval Number of Normal Distribution
}

\author{
Sha Fu*(D, Xi-Long Qu, Ye-Zhi Xiao, Hang-Jun Zhou and Guo-Bing Fan \\ School of Information Technology and Management, Hunan University of Finance and Economics, \\ Changsha 410205, China; quxilong@126.com (X.-L.Q.); xiaoyezhi0901@163.com (Y.-Z.X.); \\ zhjnudt@gmail.com (H.-J.Z.); fgb153@126.com (G.-B.F.) \\ * Correspondence: fusha15@163.com; Tel.: +86-138-7598-2792
}

Received: 6 January 2020; Accepted: 21 January 2020; Published: 8 February 2020

check for updates

\begin{abstract}
Focusing on risky decision-making problems taking the interval number of normal distribution as the information environment, this paper proposes a decision-making method based on the interval number of normal distribution. Firstly, the normalized matrix based on the decision maker's attitude is obtained through analysis and calculation. Secondly, according to the existing properties of standard normal distribution, the risk preference factors of the decision makers are considered to confirm the possibility degree of each scheme. The possibility degree is then used for establishing a possibility degree matrix and, consequently, sequencing of all schemes is conducted according to existing theories of possibility degree meaning and the value size of possibility degree. Finally, the feasibility and validity of this method is verified through calculation example analysis.
\end{abstract}

Keywords: normal distribution; interval number; risky decision-making; multi-attribute decision-making; possibility degree

\section{Introduction}

Decision-making generally exists in politics, economics, technology, and the daily life of humans. With the continuous development of social economy, decision-making with a single target and attribute is applied less and less in actual economic and management activities. In real multi-attribute decision-making, the circumstance of an uncertain natural state occurs frequently, namely risky multi-attribute decision-making. Since risky multi-attribute decision-making has an extensive practical background in the field of new product development, investment project selection, and engineering project development, the question of how to solve risky multi-attribute decision-making problems is therefore an important topic with academic research value and practical significance.

In regard to multi-attribute decision-making problems in which the attribute value is an interval number, scholars generally agree that the distribution rule of the interval number is uniform distribution [1,2], however, there are also studies [3-6] that consider the distribution rule of the interval number is normal distribution, which is more reasonable. To give a few examples, the distribution of students' examination results, the life distribution of a species, and the height distribution of people. In recent years, the research into the interval number of normal distribution has attracted the attention of experts and scholars, and has been widely used in the field of multi-attribute decision-making problems, but the research has not been mature nor perfectly optimized. For example, Liu et al. [3] analyzed the possibility measure of interval numbers, and studied the interval number complying with the normal distribution rule. They focused on the limitation that the interval number of uniform distribution was adopted to describe fuzzy evaluation value in decision-making, and proposed the method of conducting multi-attribute decision-making by applying the interval number of normal distribution. Wang and Xiao [4] provided several aggregation operators, and proposed a multi-attribute 
group decision-making method with incomplete information based on the interval number of normal distribution in group decision-making situations. Concentrating on the existing intersectional situation of two interval numbers, $\mathrm{Xu}$ and $\mathrm{Lv}$ [5] put forward the intercomparable concept of the possibility degree between interval numbers in normal distribution and a comparative method of interval numbers; according to the principle of maximum deviation, they obtained the method of ascertaining attribute weights and thus provided a multiple attribute decision-making method. Yang et al. [6] were mainly concerned with the effective supplement of incomplete information and the full utilization of uncertain information in grey number sequence prediction, and conducted the random implementation of true value to interval grey numbers of normal distribution under effective numerical coverage. Focusing on multi-attribute decision-making problems in the interval number of normal distribution, Ding and Mao [7] proposed the aggregated method for the interval number of normal distribution, established a possibility degree matrix by using possibility degree, and obtained the optimal decision by using an ordering vector method. For multi-attribute decision-making problems taking the interval number of normal distribution as the information environment, Mao et al. [8] provided the concept and related properties of cross entropy for the interval number of normal distribution, and proposed a decision-making method based on cross entropy and score function. Zhang et al. [9] used the Choquet integral to propose the normal distribution interval number for a Choquet ordered averaging operator. Chiranjibe and Madhumangal [10] attempted to lay a foundation for providing a new approach of a single-valued neutrosophic soft tool which considers many problems that contain uncertainties. In the present study, new aggregation operators of single-valued neutrosophic soft numbers have so far not yet been applied for ranking of the alternatives in decision-making problems. Song et al. [11] focused on and identified both primary strategic and operational elements that will aid managers in evaluating and making risky multi-criteria decisions on green capacity investment projects. In relevant research fields, Liu and Ren [12] only considered the deviation between membership degree and non-membership degree of the existing intuitionistic fuzzy entropy, and excluded the self-contained hesitation information in the intuitionistic fuzzy set, proposing a new class of multiple attribute decision method for intuitionistic fuzzy entropy. Shao and Zhao [13] studied the multi-criteria decision-making (MCDM) problem with completely unknown weights and evaluation information of interval-valued intuitionistic fuzzy number (IVIFN). Considering the influence of the hesitancy degree, a vector representation derived from alternative schemes and positive ideal schemes, negative ideal schemes were proposed, and a method of vector projection measure was put forward for interval intuitionistic fuzzy information. Fu et al. [14] discussed the multi-attribute decision-making (MADM) problem for attribute value with the form of IVIFN carrying incomplete attribute weight information, and comprehensively considered the correlations among attributes, proposing a decision method to address such problems. Novak Zagradjanin et al. [15] considered the multi-robot system based on the cloud technology with a high level of autonomy, which is intended for the execution of tasks in a complex and crowded environment. The proposed concept uses a multi-robot path-planning algorithm that can operate in an environment that is unknown in advance. With the aim of improving the efficiency of path planning, the implementation of multi-criteria decision-making while using the full consistency method is proposed.

In conclusion, this study considers that the attribute value is the interval number of normal distribution, and the occurrence probability of each attribute in a natural state, and in order to solve multi-attribute decision-making problems, puts forward a risky decision-making method based on the interval number of normal distribution. In theoretical research, the interval number of normal distribution is more in accordance with social and natural laws than with the interval number of uniform distribution, but it has been less researched than the interval number of uniform distribution, so the paper will help to strengthen the research in this field. In terms of practical application, the method mentioned in the paper can normalize the decision-making matrix according to different degrees of recognition of decision makers to attributes, and thus make it more widely applicable. 
The result of this study will help decision makers to make more reasonable decisions in social and economic activities, and thus bring more economic and social benefits.

\section{Theoretical Basis}

\subsection{Interval Number}

Suppose $\widetilde{a}=\left[a^{L}, a^{U}\right]=\left\{x \mid a^{L} \leq x \leq a^{U}\right\}, a^{L}, a^{U} \in R$; wherein, $a^{L}$ represents the lower limit value, $a^{U}$ represents the upper limit value, thus a closed interval in real number axis $\widetilde{a}=\left[a^{L}, a^{U}\right]$ will be referred to as the interval number. In addition, if $a^{L}=a^{U}, \widetilde{a}$ will degenerate into a certain number, namely the ordinary real number will be regarded as a special interval number [2].

Definition 1. Suppose interval number $\widetilde{a}=\left[a^{L}, a^{U}\right], \widetilde{b}=\left[b^{L}, b^{U}\right]$, if and only if $a^{L}=a^{L}, a^{U}=b^{U}$, there is $\widetilde{a}=\widetilde{b}$

Definition 2. Suppose interval number $\widetilde{a}=\left[a^{L}, a^{U}\right], \widetilde{b}=\left[b^{L}, b^{U}\right]$, if and only if $a^{L} \geq b^{U}$, there is $\widetilde{a}>\widetilde{b}$

\subsection{Interval Number of Normal Distribution}

In the multi-attribute decision-making process, the attribute value provided by decision makers is usually stable, and the attribute value tends towards a certain point, namely, an attribute value with maximum possibility. At this point, the interval number of normal distribution can be used to represent this attribute value [3].

Definition 3. Suppose $\widetilde{a}=\left[a^{L}, a^{U}\right]$ as an interval number in a set of real numbers, if the attribute value $r \in\left[a^{L}, a^{U}\right]$ complies with normal distribution $N\left(\mu_{a}, \sigma_{a}\right), \tilde{a}$ will thus be referred to as the interval number of normal distribution, wherein $\mu_{a}$ is the mean value of normal distribution and $\sigma_{a}$ is the variance of normal distribution.

According to $3 \sigma$ principle of normal distribution, $\mu_{a}$ and $\sigma_{a}$ in the above definitions can be determined by the following formula [4]:

$$
\mu_{a}=\left(a^{L}+a^{U}\right) / 2, \sigma_{a}=\left(a^{U}-a^{L}\right) / 6 .
$$

\subsection{Sequencing for the Interval Number of Normal Distribution}

For the comparison of two interval numbers of normal distribution without intersection, the sequencing can be conducted according to Definition 2, and while the intersection exists between two interval numbers of normal distribution, the sequencing can be conducted according to the following method [16]:

Theorem 1. Suppose random variable $A, B$ is intervals $\left[a^{L}, a^{U}\right],\left[b^{L}, b^{U}\right]$ respectively and complying with normal distribution, record as $A \sim N\left(\mu_{a}, \sigma_{a}\right), B \sim N\left(\mu_{b}, \sigma_{b}\right)$, thus,

$$
P(A>B)=\phi\left(\frac{\mu_{a}-\mu_{b}}{\sqrt{\sigma_{a}+\sigma_{b}}}\right)
$$

Definition 4. Suppose interval number $\widetilde{a}=\left[a^{L}, a^{U}\right], \widetilde{b}=\left[b^{L}, b^{U}\right]$, call $P(A>B)=\phi\left(\frac{\mu_{a}-\mu_{b}}{\sqrt{\sigma_{a}+\sigma_{b}}}\right)$ as possibility degree of $A>B$, wherein the value of $\phi\left(\frac{\mu_{a}-\mu_{b}}{\sqrt{\sigma_{a}+\sigma_{b}}}\right)$ is the corresponding function value of standard normal distribution $[17,18]$. 
Definition 5. If the possibility degree of the corresponding interval number $A>B$ in scheme $S_{a}, S_{b}$ is $P(A>B)=1$, then scheme $S_{a}$ is totally superior to scheme $S_{b}$, record as $S_{a}{ }_{1}^{>} S_{b}$; If the possibility degree of $A>B$ is $P(A>B)=0.5$, thus scheme $S_{a}$ is equivalent to scheme $S_{b}$, record as $S_{a}>S_{b}$. If the possibility degree of $A>B$ is $0<P(A>B)<0.5$, thus the possibility degree in which scheme $S_{a}$ is superior to scheme $S_{b}$ is $P(A>B)$, record as $S_{a} P(A>B)$ S $S_{b}$ and, at this point, the sequencing is $S_{a}<S_{b}$. If the possibility degree of $A>B$ is $0.5<P(A>B)<1$, thus the possibility degree in which scheme $S_{a}$ is superior to scheme $S_{b}$ is $P(A>B)$, record as $S_{a}>(A>B)$ S $S_{b}$ and, at this point, the sequencing is $S_{a}>S_{b}[19,20]$.

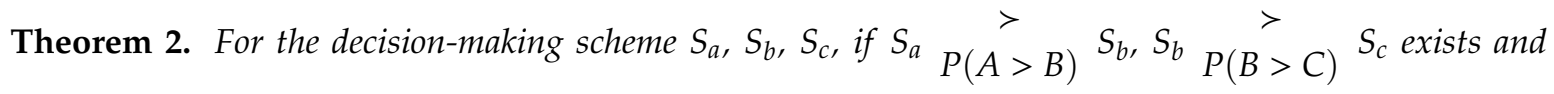

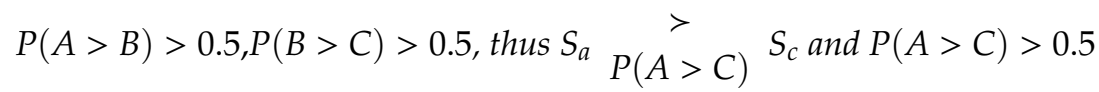

Through Definition 5 and Theorems 1 and 2, the sequencing method for the interval number of normal distribution can be obtained, namely, the possibility degree matrix with pairwise comparison can be established by a pairwise comparison of all interval numbers of normal distribution.

$$
P=\left[\begin{array}{cccc}
p_{11} & p_{12} & \cdots & p_{1 m} \\
p_{21} & p_{22} & \cdots & p_{2 m} \\
\vdots & \vdots & \ddots & \vdots \\
p_{m 1} & p_{m 2} & \cdots & p_{m m}
\end{array}\right]
$$

wherein $P_{i j}$ represents the possibility degree of $S_{i}>S_{j}$, if $P_{i j}>0.5$, scheme $i$ will be superior to scheme $j$ and, otherwise, scheme $j$ will be superior to scheme $i$ [21].

\section{Risky Multi-Attribute Decision-Making Method}

\subsection{Problem Description}

Consider a certain risky multi-attribute decision-making problem, as a matter of convenience, record as $A=\left\{A_{1}, A_{2}, \cdots, A_{m}\right\}$ which represents the set of $m$ alternative schemes, wherein $A_{i}$ represents the alternative scheme of number $i ; C=\left\{C_{1}, C_{2}, \cdots, C_{n}\right\}$ represents the set of number $n$ attributes, wherein $C_{j}$ represents the attribute of number $j ; w=\left\{w_{1}, w_{2}, \cdots, w_{n}\right\}$ represents the weight vector of the attribute, wherein $w_{j}$ is the weight or importance degree of attribute $C_{j}$ which meets $w_{j} \geq 0$ and $\sum_{j=1}^{n} W_{j}=1 ; \theta=\left\{\theta_{1}, \theta_{2}, \cdots, \theta_{h}\right\}$ represents the set of natural states, wherein $\theta_{t}$ represents the state of number $t$ and $p_{t}$ represents the occurrence possibility of state $\theta_{t}$, which meets $p_{t} \geq 0$ and $\sum_{t=1}^{h} p_{t}=1$.

Suppose the interval number $r_{x y z}=\left[r_{x y z}^{L}, r_{x y z}^{U}\right]$ represents the attribute value in natural state $\theta_{z}$ for scheme $A_{x}$ in allusion to attribute $C_{y}$, thus the risk decision-making matrix $R$ is established [22].

\subsection{Normalization of Decision-Making Matrix}

In solving risky multi-attribute decision-making problems, generally, the attributes can be divided into benefit-type and cost-type, where the bigger benefit-type attribute value will be better, and the smaller cost-type attribute value will be better. When both appear in a decision-making process at the same time, in order to eliminate the influence on decision-making results caused by different dimensions of different attributes, the normalization of each attribute value should be processed. There are numerous methods for the normalization of the attribute values of interval values, and each has its own pertinence, for example, the extreme value in the range transformation method significantly 
influences the specification results. This study adopts the range transformation method to process the normalization of the decision-making matrix, and the specific normalization formulas are shown below.

Benefit-type attribute:

$$
a_{x y z}^{L}=\frac{r_{x y z}^{L}-\operatorname{minr}_{x y z}}{\operatorname{maxr}_{x} r_{x z}-\min _{x} r_{x y}}, a_{x y z}^{U}=\frac{r_{x y z}^{U}-\operatorname{minr}_{x y z}}{\max _{x} r_{x y z}-\operatorname{minr}_{x} r_{x z}}
$$

Cost-type attribute:

$$
a_{x y z}^{L}=\frac{\operatorname{maxr}_{x y z}-r_{x y z}^{U}}{\max _{x} r_{x y z}-\operatorname{minr}_{x} r_{x y z}}, a_{x y z}^{U}=\frac{\operatorname{maxr}_{x y z}-r_{x y z}^{L}}{\max _{x} r_{x y z}-\operatorname{minr}_{x y z}}
$$

wherein $\max _{x} r_{x y z}=\max \left(\max _{x} r_{x y z}^{L}, \max _{x} r_{x y z}^{U}\right) ; \min _{x} r_{x y z}=\min \left(\operatorname{minr}_{x}^{L} \min _{x}, \min _{x y z}^{U}\right)$.

Concentrating on risky multi-attribute decision-making problems, in view of the limitations of common normalization methods, the normalization result can be amended according to the preference of the decision makers. According to Formula (5), transform the results of the above steps again and obtain the normalization results:

$$
b_{x y z}^{L}=\exp \left[\left(a_{x y z}^{L}-1\right) / k_{i}\right], b_{x y z}^{U}=\exp \left[\left(a_{x y z}^{U}-1\right) / k_{i}\right]
$$

wherein the value of $k_{i}$ is based on the judgment of decision makers, take $k_{i}=1,2,3$, and the value of $i$ is the sequence number of attribute $C_{j}, 1 \leq \mathrm{i} \leq \mathrm{n}$. If the value of $k_{i}$ is bigger, the degree of recognition for its corresponding attribute will be lower $[23,24]$.

\subsection{Decision-Making Steps}

In regard to the above problem, the specific steps of the risky multi-attribute decision-making method based on the interval number of normal distribution mentioned in the paper are as follows:

Step 1 According to Formulas (3) and (4), conduct the normalization to risky decision-making matrix by using the range transformation method, and obtain the decision-making matrix $R$.

Step 2 In view of the limitations of the common normalization method, according to Formula (5), transform the attribute value again based on the preference of the decision makers, and obtain the decision-making matrix $R_{1}$.

Step 3 For the decision-making matrix after normalization, according to Formula (6), conduct weighting operation to attribute value through occurrence probability $P_{k}$ in different natural states $\theta_{k}$, obtain decision-making matrix $R_{2}=\left(r_{x y}^{L}, r_{x y}^{U}\right)$ [25].

$$
r_{x y}^{L}=\sum_{k=1}^{m} r_{x y z}^{L} p_{k}, r_{x y}^{U}=\sum_{k=1}^{m} r_{x y z}^{U} p_{k}
$$

Step 4 Combine with the weight vector of attribute $w$, calculate the deviation $V_{x}$ of each scheme according to Formula (7).

$$
V_{x}=\sum_{y=1}^{m} w_{y} r_{x y}=\left[\sum_{y=1}^{m} w_{y} r_{x y}^{L}, \sum_{y=1}^{m} w_{y} r_{x y}^{U}\right]
$$

Step 5 According to the sequencing method for the interval value of normal distribution derived from the above steps, establish the possibility degree matrix of pairwise comparison $P$, and hereafter, conduct the sequencing of all schemes. 


\section{Calculating Example Analysis}

Consider a selection problem of a new product development project [1]. A company proposes to develop an electronic product; where there are 5 schemes $\left(A_{1}, A_{2}, \cdots, A_{5}\right)$ available, the main attributes to be considered include developing $\operatorname{cost} C_{1}$, sales quality of product $C_{2}$, and rate of return $C_{3}$. In these three attributes, $C_{1}$ is cost-type attribute, $C_{2}$ and $C_{3}$ are benefit-type attributes, and the attribute values of all attributes are interval numbers. Suppose the value located in the interval complies with normal distribution, furthermore, $\left(\theta_{1}, \theta_{2}, \theta_{3}\right)$ exist in the future market environment, representing excellent, average and poor states respectively, and their probability of occurrence is $p=(0.3,0.4,0.3)$ respectively. Suppose the attribute weight vector provided by the decision makers is $w=(0.35,0.25,0.4)^{T}$, the risky decision-making matrix is shown in Table 1.

Table 1. Risky Decision-Making Matrix.

\begin{tabular}{cccccccccc}
\hline \multirow{3}{*}{ Scheme } & \multicolumn{3}{c}{ C1 } & \multicolumn{3}{c}{ C2 } & \multicolumn{3}{c}{ C3 } \\
\cline { 2 - 10 } & $\boldsymbol{\theta}_{1}$ & $\boldsymbol{\theta}_{2}$ & $\boldsymbol{\theta}_{3}$ & $\boldsymbol{\theta}_{1}$ & $\boldsymbol{\theta}_{2}$ & $\boldsymbol{\theta}_{3}$ & $\boldsymbol{\theta}_{1}$ & $\boldsymbol{\theta}_{2}$ & $\boldsymbol{\theta}_{3}$ \\
\hline A1 & {$[80,90]$} & {$[90,100]$} & {$[90,110]$} & {$[100,120]$} & {$[80,100]$} & {$[70,80]$} & {$[12,16]$} & {$[9,12]$} & {$[6,8]$} \\
A2 & {$[90,100]$} & {$[100,110]$} & {$[110,120]$} & {$[110,120]$} & {$[90,100]$} & {$[80,90]$} & {$[12,18]$} & {$[10,15]$} & {$[7,10]$} \\
A3 & {$[90,110]$} & {$[100,120]$} & {$[110,130]$} & {$[120,130]$} & {$[100,110]$} & {$[80,100]$} & {$[15,22]$} & {$[13,20]$} & {$[8,12]$} \\
A4 & {$[100,110]$} & {$[110,130]$} & {$[120,130]$} & {$[100,110]$} & {$[80,90]$} & {$[60,80]$} & {$[18,23]$} & {$[15,20]$} & {$[6,10]$} \\
A5 & {$[110,120]$} & {$[115,130]$} & {$[120,140]$} & {$[120,150]$} & {$[100,120]$} & {$[90,100]$} & {$[20,25]$} & {$[12,18]$} & {$[8,10]$} \\
\hline
\end{tabular}

(1) According to Formulas (3) and (4), the range transformation method is adopted for processing the normalization of risky decision-making matrix to get decision-making matrix $R$, shown in Table 2 .

Table 2. Risky Decision-Making Matrix $R$ after Normalized Processing.

\begin{tabular}{|c|c|c|c|c|c|c|c|c|c|}
\hline \multirow{2}{*}{ Scheme } & \multicolumn{3}{|c|}{$\mathrm{C} 1$} & \multicolumn{3}{|c|}{$\mathrm{C} 2$} & \multicolumn{3}{|c|}{ C3 } \\
\hline & $\theta_{1}$ & $\theta_{2}$ & $\theta_{3}$ & $\theta_{1}$ & $\theta_{2}$ & $\theta_{3}$ & $\boldsymbol{\theta}_{1}$ & $\theta_{2}$ & $\theta_{3}$ \\
\hline A1 & {$[0.75,1]$} & {$[0.75,1]$} & {$[0.6,1]$} & {$[0,0.4]$} & {$[0,0.5]$} & {$[0.25,0.5]$} & {$[0,0.308]$} & {$[0,0.273]$} & {$[0,0.333]$} \\
\hline A3 & {$[0.25,0.75]$} & {$[0.25,0.75]$} & {$[0.2,0.6]$} & {$[0.4,0.6]$} & {$[0.5,0.75]$} & {$[0.5,1]$} & {$[0.231,0.769]$} & {$[0.364,1]$} & {$[0.333,1]$} \\
\hline $\mathrm{A} 4$ & {$[0.25,0.5]$} & {$[0,0.5]$} & {$[0.2,0.4]$} & {$[0,0.2]$} & {$[0,0.25]$} & {$[0,0.5]$} & {$[0.462,846]$} & {$[0.545,1]$} & {$[0,0.667]$} \\
\hline A5 & {$[0,0.25]$} & {$[0,0.375]$} & {$[0,0.4]$} & {$[0.4,1]$} & {$[0.5,1]$} & {$[0.75,1]$} & {$[0.615,1]$} & {$[0.273,0.818]$} & {$[0.333,0.667]$} \\
\hline
\end{tabular}

(2) According to Formula (5), transform attribute value again based on the preferences of the decision makers, suppose decision makers attach importance to each attribute comparably, take $k_{1}=1$, $k_{2}=1, k_{3}=1$, and obtain the decision-making matrix $R_{1}$, shown in Table 3 .

Table 3. Risky Decision-Making Matrix $R_{1}$ after Transforming Again.

\begin{tabular}{|c|c|c|c|c|c|c|c|c|c|}
\hline \multirow{2}{*}{ Scheme } & \multicolumn{3}{|c|}{$\mathrm{C} 1$} & \multicolumn{3}{|c|}{$\mathrm{C} 2$} & \multicolumn{3}{|c|}{$\mathrm{C} 3$} \\
\hline & $\theta_{1}$ & $\theta_{2}$ & $\theta_{3}$ & $\boldsymbol{\theta}_{1}$ & $\theta_{2}$ & $\theta_{3}$ & $\boldsymbol{\theta}_{1}$ & $\theta_{2}$ & $\theta_{3}$ \\
\hline A1 & {$[0.779,1.000]$} & {$[0.779,1.000]$} & {$[0.670,1.000]$} & {$[0.368,0.549]$} & {$[0.368,0.607]$} & {$[0.472,0.607]$} & {$[0.368,0.500]$} & {$[0.368,0.0 .483]$} & {$[0.368,0.513]$} \\
\hline $\mathrm{A} 3$ & {$[0.472,0.779]$} & {$[0.472,0.779]$} & {$[0.449,0.670]$} & {$[0.549,0.670]$} & {$[0.607,0.779]$} & {$[0.607,1.000]$} & {$[0.463,0.794]$} & {$[0.529,1.000]$} & {$[0.513,1.000]$} \\
\hline A4 & {$[0.472,0.607]$} & {$[0.368,0.607]$} & {$[0.449,0.549]$} & {$[0.368,0.449]$} & {$[0.368,0.472]$} & {$[0.368,0.607]$} & {$[0.584,0.857]$} & {$[0.635,1.000]$} & {$[0.368,0.717]$} \\
\hline A5 & {$[0.368,0.472]$} & {$[0.368,0.535]$} & {$[0.368,0.549]$} & {$[0.549,1.000]$} & {$[0.607,1.000]$} & {$[0.779,1.000]$} & {$[0.681,1.000]$} & {$[0.483,0.834]$} & {$[0.513,0.717]$} \\
\hline
\end{tabular}

(3) According to Formula (6), conduct the weighting operation to attribute value through the probability $p_{k}$ in every natural state $\theta_{k}$, and then obtain the decision-making matrix $R_{2}$.

$$
\mathrm{R}_{2}=\left[\begin{array}{ccc}
{[0.746,1.000]} & {[0.399,0.589]} & {[0.368,0.497]} \\
{[0.589,0.746]} & {[0.506,0.641]} & {[0.402,0.644]} \\
{[0.465,0.746]} & {[0.589,0.813]} & {[0.505,0.938]} \\
{[0.424,0.589]} & {[0.368,0.506]} & {[0.539,0.872]} \\
{[0.368,0.520]} & {[0.641,1.000]} & {[0.552,0.848]}
\end{array}\right]
$$


(4) Combine the known attribute weight vector $w=(0.35,0.25,0.4)^{T}$, and calculate deviation of each scheme $V_{x}$ according to Formula (7).

$$
\begin{gathered}
V_{1}=[0.508,0.696], V_{2}=[0.493,0.679], V_{3}=[0.512,0.840], \\
V_{4}=[0.456,0.682], V_{5}=[0.510,0.772],
\end{gathered}
$$

According to Formula (1), transform deviation $V_{x}$ to the form of $\left(\mu_{a}, \sigma_{a}\right)$.

$$
\begin{gathered}
V_{1}^{\prime}=[0.602,0.031], V_{2}^{\prime}=[0.586,0.031], V_{3}^{\prime}=[0.676,0.055], \\
V_{4}^{\prime}=[0.569,0.038], V_{5}^{\prime}=[0.641,0.044],
\end{gathered}
$$

(5) According to the sequencing method for the interval number of normal distribution and theorem 2, a possibility degree matrix with pairwise comparison $P$ can be established, and hereby, all schemes can be sequenced.

$$
\mathrm{P}=\left[\begin{array}{lllll}
0.5000 & 0.5255 & 0.4013 & 0.5505 & 0.4443 \\
0.4745 & 0.5000 & 0.3783 & 0.5267 & 0.4207 \\
0.5987 & 0.6217 & 0.5000 & 0.6380 & 0.5450 \\
0.4495 & 0.4733 & 0.3620 & 0.5000 & 0.4013 \\
0.5557 & 0.5793 & 0.4550 & 0.5987 & 0.5000
\end{array}\right]
$$

From this, the sequencing result of each scheme is $A_{3}>A_{5}>A_{1}>A_{2}>A_{4}$, therefore, when the value of $k_{i}$ is 1 , Scheme $A_{3}$ will be optimal.

(6) In order to verify the influence on decision-making result caused by parameter $k_{i}$, suppose the corresponding values of three attributes are $k_{1}, k_{2}, k_{3}$ respectively, now the sequencing result of each scheme is shown in Table 4.

Table 4. Influence on Decision-Making Result Caused by $k_{i}$ Value.

\begin{tabular}{cccc}
\hline & $\boldsymbol{k}_{\boldsymbol{i}}$ Value & & Sequencing Result of Each Scheme \\
\hline $\mathrm{K} 1=1$ & $\mathrm{~K} 2=1$ & $\mathrm{~K} 3=1$ & $\mathrm{~A} 3>\mathrm{A} 5>\mathrm{A} 1>\mathrm{A} 2>\mathrm{A} 4$ \\
$\mathrm{~K} 1=1$ & $\mathrm{~K} 2=1$ & $\mathrm{~K} 3=3$ & $\mathrm{~A} 3>\mathrm{A} 5>\mathrm{A} 1>\mathrm{A} 4>\mathrm{A} 2$ \\
$\mathrm{~K} 1=1$ & $\mathrm{~K} 2=3$ & $\mathrm{~K} 3=1$ & $\mathrm{~A} 3>\mathrm{A} 5>\mathrm{A} 1>\mathrm{A} 4>\mathrm{A} 2$ \\
$\mathrm{~K} 1=1$ & $\mathrm{~K} 2=3$ & $\mathrm{~K} 3=3$ & $\mathrm{~A} 3>\mathrm{A} 5>\mathrm{A} 1>\mathrm{A} 2>\mathrm{A} 4$ \\
$\mathrm{~K} 1=3$ & $\mathrm{~K} 2=1$ & $\mathrm{~K} 3=1$ & $\mathrm{~A} 5>\mathrm{A} 3>\mathrm{A} 2>\mathrm{A} 1>\mathrm{A} 4$ \\
$\mathrm{~K} 1=3$ & $\mathrm{~K} 2=1$ & $\mathrm{~K} 3=3$ & $\mathrm{~A} 5>\mathrm{A} 3>\mathrm{A} 2>\mathrm{A} 1>\mathrm{A} 4$ \\
$\mathrm{~K} 1=3$ & $\mathrm{~K} 2=3$ & $\mathrm{~K} 3=1$ & $\mathrm{~A} 3>\mathrm{A} 5>\mathrm{A} 2>\mathrm{A} 4>\mathrm{A} 1$ \\
$\mathrm{~K} 1=3$ & $\mathrm{~K} 2=3$ & $\mathrm{~K} 3=3$ & $\mathrm{~A} 3>\mathrm{A} 1>\mathrm{A} 2>\mathrm{A} 4$ \\
\hline
\end{tabular}

According to the decision-making method considering regret aversion mentality in Reference [1], its final sequencing result is the same as the result of this research, in which $k_{1}=3, k_{2}=3$, and $k_{3}=1$. From the above table, when decision makers are more concerned about cost and rate of return, Scheme 3 will be superior to Scheme 5; when decision makers are more concerned about sales volume and rate of return, Scheme 5 will be superior to Scheme 3.

The characteristics of the method mentioned in [1] are that it considers the regret aversion mentality and behavior of decision makers and obtains the sequencing result by calculating the utility value of the attribute as well as the regret and delight values between schemes. From Table 4, it can be seen that this method tends toward scheme $A_{3}$ with lower cost and higher rate of return; for scheme $A_{5}$ with higher cost and rate of return, its sequencing result is lower due to higher risk and regret aversion mentality. From original data, it can be seen that both the cost and the sales value of Scheme 5 are higher than those of Scheme 3. In conclusion, the optimal scheme using method in this reference tends to be conservative. 
The method mentioned in this paper can be adapted to the requirements of various decision makers for decision making, since $k_{i}$ can take different values. Conservative decision makers can take a smaller corresponding value of cost-type attribute $k_{i}$, while optimistic decision makers can take a smaller corresponding value of benefit-type attribute $k_{i}$. Therefore, the decision-making method mentioned in this paper is closer to actual production and life in the application of multi-attribute decision-making problems, can serve decision makers with different personalities, and can be more widely applied.

\section{Conclusions}

For interval number multi-attribute decision-making problems, the interval number of normal distribution undoubtedly has great practical application value, since the distribution rule of interval numbers receives less attention. The paper utilizes the basic theory of normal distribution, transforms the sequencing of decision-making schemes into the comparison of possibility degrees, and thus obtains the optimal decision after normalizing the initial matrix based on the attitude of decision makers. As a result, it provides a multi-attribute decision-making approach based on the interval number of normal distribution and verifies the feasibility and effectiveness of the aforementioned methods by combining calculation example analysis, and enriches the application of decision-making methods [26]. The method mentioned in this study is appropriate for fuzzy decision-making environments in view of its superiority in a number of respects. Not only does the interval number of normal distribution describe fuzzy evaluation value in comparison to the interval value of uniform distribution but, furthermore, the decision-making problems described by the interval number of normal distribution are closer to real life, and the influence on decision-making behavior caused by risk preference factors is taken into full consideration. Therefore, this method can be widely applied, and has both promotional and actual decision-making value.

Author Contributions: All authors have contributed to this research equally. All authors have read and agreed to the published version of the manuscript.

Funding: This work was supported in part by the Scientific Research Fund of Hunan Provincial Education Department under Grant 17B046 and 18A441, in part by the Hunan Provincial Natural Science Foundation of China under Grant 2016JJ4012 and 2017JJ2016, and in part by the Hunan Education Science "13th Five-Year" plan special topic of Party Building Research under Grant XJK18DJA1.

Conflicts of Interest: The authors declare no conflicts of interest.

\section{References}

1. Zhang, X.; Fan, Z.-P.; Chen, F.-D. Risky Multiple Attribute Decision Making with Regret Aversion. J. Syst. Manag. 2014, 23, 111-117.

2. Su, Z.-X.; Wang, L.; Xia, G.-P. Extended VIKOR method for dynamic multi-attribute decision making with interval numbers. Control Decis. 2010, 25, 836-840, 846.

3. Liu, L.; Chen, Y.-X.; Ge, Z.-H. Probability measure of interval-number based on normal distribution and multi-attribute decision making. Syst. Eng. Electron. 2008, 30, 652-654.

4. Wang, X.-F.; Xiao, M.-S. Approach of group decision making based on normal distribution interval number with incomplete information. Control Decis. 2010, 25, 1494-1498.

5. Xu, G.-L.; Lv, Y.-J. Approach of Multi-attribute Decision-making Based on Normal Distribution Interval Number. Syst. Eng. 2011, 29, 120-123.

6. Yang, J.-W.; Xiao, X.-P.; Guo, J.-H. Gray prediction model of normal distribution interval grey number. Control Decis. 2015, 30, 1711-1716.

7. Ding, D.-H.; Mao, J.-J.M. Inclusion degree and possibility degree of normal distribution interval number and their application. J. Hefei Univ. Technol. 2013, 36, 1136-1139.

8. Mao, J.-J.; Wang, C.-C.; Yao, D.-B.; Sun, L. Multi-attribute decision-making method of normal distribution interval number based on cross-entropy. Comput. Eng. Appl. 2012, 48, 44-48. 
9. Zhang, B.; Yu, Z.-W.; Shao, J.-S. Research on the Comprehensive Evaluation in Enterprise Operation Environment with Normal Distribution Interval Number. J. Comput. Theor. Nanosci. 2016, 13, 7342-7346. [CrossRef]

10. Jana, C.; Pal, M. A Robust Single-Valued Neutrosophic Soft Aggregation Operators in Multi-Criteria Decision Making. Symmetry 2019, 11, 110. [CrossRef]

11. Song, Y.; Yao, S.; Yu, D.-H.; Shen, Y. Risky multi-criteria group decision making on green capacity investment projects based on supply chain. J. Bus. Econ. Manag. 2017, 18, 355-372. [CrossRef]

12. Liu, M.-F.; Ren, H.-P. Intuitionistic fuzzy multi-attribute decision making based on a new intuitionistic fuzzy entropy measure. Syst. Eng. Theory Pract. 2015, 35, 1-8.

13. Shao, L.-S.; Zhao, L.-L. Bidirectional projection method with interval-valued intuitionistic fuzzy information. Control Decis. 2016, 31, 571-576.

14. Fu, S.; Qu, X.-L.; Zhou, H.-J.; Fan, G.-B. A Multi-Attribute Decision-Making Model Using Interval-valued Intuitionistic Fuzzy Numbers and Attribute Correlation. Int. J. Enterp. Inf. Syst. 2018, 14, 21-34. [CrossRef]

15. Zagradjanin, N.; Pamucar, D.; Jovanovic, K. Cloud-Based Multi-Robot Path Planning in Complex and Crowded Environment with Multi-Criteria Decision Making Using Full Consistency Method. Symmetry 2019, 11, 1241. [CrossRef]

16. Gitinavard, H.; Pishvaee, M.S.; Jalalvand, F. A hierarchical multi-criteria group decision-making method based on TOPSIS and hesitant fuzzy information. Int. J. Appl. Decis. Sci. 2017, 10, 213-232.

17. Liu, B.-S.; Shen, Y.-H.; Zhang, W.; Chen, X.-H.; Wang, X.-Q. An interval-valued intuitionistic fuzzy principal component analysis model-based method for complex multi-attribute large-group decision-making. Eur. J. Oper. Res. 2015, 245, 209-225. [CrossRef]

18. Xu, Z.-S. Intuitionistic Fuzzy Information Aggregation Theory and Application; Science Press: Beijing, China, 2016; pp. $45-67$.

19. Xiao, J.; Zhang, Y.; Fu, C. Comparison between Methods of Interval Number Ranking Based on Possibility. J. Tianjin Univ. 2011, 44, 705-711.

20. Liu, P.-D.; Xia, J.-T.; Chi, P.-P. A method of multi-attribute decision making under risk based on interval probability. J. Intell. Fuzzy Syst. 2014, 26, 3005-3011. [CrossRef]

21. Qin, J.-D.; Liu, X.-W.; Pedrycz, W. Multi-attribute group decision making based on Choquet integral under interval-valued intuitionistic fuzzy environment. Int. J. Comput. Intell. Syst. 2016, 9, 133-152. [CrossRef]

22. Temur, G.T. A novel multi attribute decision making approach for location decision under high uncertainty. Appl. Soft Comput. 2016, 40, 674-682. [CrossRef]

23. Li, C.-B.; Yuan, J.-H. A New Multi-attribute Decision-Making Method with Three-Parameter Interval Grey Linguistic Variable. Int. J. Fuzzy Syst. 2017, 19, 292-300. [CrossRef]

24. Harsh, S.; Deb, D.D.; Muresan, V.; Unguresan, M. Multi-Criteria Decision Making Approach for Hybrid Operation of Wind Farms. Symmetry 2019, 11, 675.

25. Sureeyatanapas, P.; Pathumnakul, S. Impacts of using relative weights in multiple criteria decision making: A comparative study between independent- and overlapping-criteria decision problems. Int. J. Appl. Decis. Sci. 2017, 10, 101-117.

26. Nuchkrua, T.; Leephakpreeda, T. Fuzzy Self-Tuning PID Control of Hydrogen-Driven Pneumatic Artificial Muscle Actuator. J. Bionic Eng. 2013, 10, 329-340. [CrossRef]

(C) 2020 by the authors. Licensee MDPI, Basel, Switzerland. This article is an open access article distributed under the terms and conditions of the Creative Commons Attribution (CC BY) license (http://creativecommons.org/licenses/by/4.0/). 